\title{
On the Influence of Heart Rate and Coupling Interval Prematurity on Heart Rate Turbulence
}

\author{
Óscar Barquero-Pérez, Carlos Figuera, Rebeca Goya-Esteban, Inmaculada Mora-Jiménez, \\ F. Javier Gimeno-Blanes, Pablo Laguna, Senior Member, IEEE, Juan Pablo Martínez, Eduardo Gil, \\ Leif Sörnmo, Senior Member, IEEE, Arcadi García-Alberola, José Luis Rojo-Álvarez, Senior Member, IEEE
}

\begin{abstract}
Objective: Heart rate turbulence (HRT) has been successfully explored for cardiac risk stratification. While HRT is known to be influenced by the heart rate (HR) and the coupling interval (CI), non-concordant results have been reported on how the CI influences HRT. The purpose of this study is to investigate HRT changes in terms of CI and HR by means of a specially designed protocol. Methods: A dataset was acquired from 11 patients with structurally normal hearts for which CI was altered by different pacing trains and $\mathrm{HR}$ by isoproterenol during electrophysiological study (EPS). The protocol was designed so that, first, the effect of HR changes on HRT, and, second, the combined effect of $\mathrm{HR}$ and CI could be explored. As a complement to the EPS dataset, a database of 24-h Holters from 61 acute myocardial infarction (AMI) patients was studied for the purpose of assessing risk. Data analysis was performed by using different nonlinear ridge regression models, and the relevance of model variables was assessed using resampling methods. The EPS subjects, with and without isoproterenol, were analyzed separately. Results: The proposed nonlinear regression models were found to account for the influence of HR and CI on HRT, both in patients undergoing EPS without isoproterenol and in low-risk AMI patients, whereas this influence was absent in highrisk AMI patients. Moreover, model coefficients related to CI were not statistically significant, $p>0.05$, on EPS subjects with isoproterenol. Conclusion: The observed relationship between CI and HRT, being in agreement with the baroreflex hypothesis, was statistically significant $(p<0.05)$, when decoupling the effect of HR and normalizing the CI by the HR. Significance: The results of this work can help to provide new risk indicators that take into account physiological influence on HRT, as well as to model how this influence changes in different cardiac conditions.
\end{abstract}

Index Terms-Heart Rate Turbulence, Coupling Interval, Heart Rate, Electrophysiological Study, Myocardial Infarction.

\section{INTRODUCTION}

$\mathbf{H}$ EART rate turbulence (HRT) is the physiological heart rate response to a spontaneous ventricular premature contraction (VPC). In normal subjects, this response consists of an initial acceleration and a subsequent deceleration in heart

OBP, CF, RGB, IMJ and JLRA are with the Signal Theory and Communications, Telematics and Computing Department, Rey Juan Carlos University, Madrid, Spain (e-mail to \{oscar.barquero@urjc.es\}. AGA is with the Arrhythmia Unit, Hospital Universitario Virgen de la Arrixaca, Murcia, Spain. JGB is with the Signal Theory and Communications Department, Miguel HernÃąndez University, Elche, Spain. PL, JPM and EG are with Biomedical Signal Interpretation and Computational Simulation Group (BSICoS), Aragón Institute of Engineering Research (I3A), IIS Aragón, Universidad de Zaragoza, Spain, and Centro de Investigación Biomédica en Red en Bioingeniería, Biomateriales y Nanomedicina (CIBERBBN), Zaragoza, Spain. LS is with Department of Biomedical Engineering and Center of Integrative Electrocardiology, Lund University, Lund, Sweden.

Manuscript received xxx xx, 2016; revised xxxx xx, 2016. rate (HR). Its absence has been shown to be a powerful risk predictor of cardiovascular events following acute myocardial infarction (AMI) and other cardiac conditions [1], [2]. HRT is usually characterized by turbulence onset (TO) and turbulence slope (TS) parameters. TO represents the degree of sinus acceleration following a VPC, and is defined by the relative difference of the averages of the two normal RR intervals before and after the VPC. TS represents the rate of sinus deceleration after the initial acceleration, and is defined as the maximum slope of the linear regression of every five consecutive RR-intervals within the first 15 RR-intervals following the VPC (VPC tachogram) [2], [3].

The baroreflex hypothesis of HRT origin states that increased prematurity of the VPC causes a larger drop in blood pressure, which, in turn, leads to a stronger HRT response. [2]. The standard approach to assessing HRT uses signal averaging of all the available isolated VPC tachograms, from which TS and $\mathrm{TO}$ are computed. While signal averaging improves the signal-to-noise ratio (SNR), it could also mask the influence of different physiological factors on HRT, especially when these factors are not independent [4], [5]. Several physiological factors modulate HRT, namely, HR, VPC prematurity, and circadianity [6], [7], [8]. However, existing studies present surprisingly contradictory results which, sometimes, are in conflict with the baroreflex hypothesis.

The aim of this work is to assess the interaction of coupling interval (CI) and HR with HRT. Instead of using signal averaging, HRT was measured by computing TS on each individual VPC tachogram. For this purpose, data were acquired from patients with structurally normal hearts who underwent electrophysiological study (EPS). A clinical protocol was specifically designed so that the CI was controlled with a programmed cardiac pacing protocol, and the HR was controlled using isoproterenol. The electrophysiological (EP) protocol consisted of two substudies: one for analyzing the relationship between HR and HRT, and another for analyzing the combined effect of HR and CI on HRT. We also analyzed these interactions by using Holter recordings from patients after an AMI episode, so that EPS data, acquired during favorable conditions, could be compared to data from ambulatory monitoring where much higher noise levels are encountered. Suitable regression models were used to replicate previous results [9], [10], [11], [12], and to explain the combined influence of CI and HR on HRT.

The structure of the paper is as follows. In Section II, the EPS protocol and the Holter database are described. In

Copyright (c) 2016 IEEE. Personal use of this material is permitted. However, permission to use this material for any

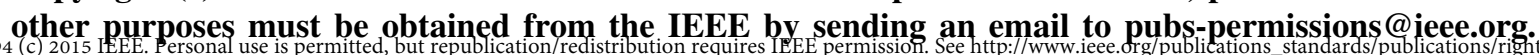


Section III, the analysis methods and the nonlinear ridge regression models are presented, as well as the statistical method for benchmarking different models. In Section IV, statistical analysis and results are presented. In Section V, the present results are discussed and related to existing results in the literature. Conclusions of this work are presented in Section VI.

\section{Clinical Datasets}

\section{A. EPS Protocol}

Eleven patients $(50 \pm 15$ years, 7 women) with structurally normal hearts were included in the study, all of them referred for EPS in the Hospital Universitario Virgen de la Arrixaca (Murcia, Spain). The study was approved by the local Ethics Committee and all participants granted a signed informed consent. The EPS was performed during sinus rhythm after ablation procedures, and sequences of 10 single induced VPCs were delivered every $20 \mathrm{~s}$ from the right ventricular apex.

The study was structured into a fixed CI protocol (5 patients) and a variable CI protocol (6 patients), designed to investigate the influence of HR on HRT, and the combined influence of HR and CI on HRT, respectively. The HR was increased with isoproterenol, which does so by activating beta-1 adrenergic receptors in the heart [13]. Isoproterenol, $0.8 \mu \mathrm{g} / \mathrm{ml}$, was delivered as a continuous infusion via a cannulated antecubital vein. The initial rate of infusion was $30 \mathrm{ml} / \mathrm{h}$ and then increased in increments of $10 \mathrm{ml} / \mathrm{h}$ every 2 minutes until the target HR of 20-30\% above baseline was achieved.

Fixed CI protocol - Influence of HR on TS (5 patients). The purpose of this protocol was to assess the influence of HR on TS, under the assumption that high HR (short sinus cycle length, SCL) produces a lower TS. There were two phases in this protocol, a control phase and an isoproterenol infusion phase. VPCs were delivered with a prematurity of $70 \%$ of the baseline SCL as measured at the start of each phase.

Variable CI protocol - Influence of HR and prematurity on TS (6 patients). The purpose of this protocol was twofold: First, to verify CI modulation of TS (under the assumption that a shorter CI is associated with more pronounced HRT); Second, to verify the possible interaction between HR and CI suggested in [14]. The variable CI protocol was also delivered in two phases, the control phase and the isoproterenol phase. VPCs were delivered with an initial prematurity of $95 \%$ of the baseline SCL, as measured at the start of each phase, after which prematurity was decremented by $70 \mathrm{~ms}$ each time until the extrastimulus was no longer captured. Note that the variable CI protocol aimed to decouple the effect of $\mathrm{HR}$ and CI on TS modulation, by considering two scenarios, one with low HR (long SCL, control phase) and one with high HR (short SCL, isoproterenol phase). CI was modified to evaluate the isolated influence of VPC prematurity.

Both phases were repeated twice for each protocol in order to obtain enough valid VPC tachograms.

\section{B. AMI Dataset}

The AMI dataset consisted of recordings from 61 AMI patients (64 \pm 9 years, 18 women) who underwent emergency coronary angiography, and, when appropriate, percutaneous coronary intervention. These data were collected in a prospective study at University Hospital Virgen de la Arrixaca [15] in order to evaluate the impact of primary angioplasty on the indication for implantable defibrillator in patients with AMI. 24-h ambulatory electrocardiographic monitoring was done in patients with stable sinus rhythm between 2 and 6 weeks after the infarction, and patients with at least 1 VPC during the monitoring period were included in the study.

There were 58 patients with at least 1 VPC. The patients were split 3 ways according to their risk for mortality according to standard HRT classification, high risk (TS $<2.5 \mathrm{~ms} / \mathrm{RR}$ interval and TO $>0 \%$ ), low risk (TS $>2.5$ and TO $<0$ ), and moderate risk (TS $<2.5$ exclusive or TO $>0$ ) [2], of which the first two subsets were employed. The low risk subset was comprised of 17 patients ( $63 \pm 12$ years, 5 women) and the high risk subset was comprised of 6 patients $(70 \pm 6$ years, 1 woman). The purpose of the splitting was to compare the low risk subset with the EPS patients and then to contrast it with the high risk subset.

\section{REgRESSION MODELING OF THE HRT}

We propose to use a data-driven model of HRT as a function of HR and CI for analyzing the hypothesis that TS can be partially explained as a nonlinear function of $\mathrm{HR}$ and CI. A nonlinear ridge regression model is considered, which accounts for linear and nonlinear interactions of $\mathrm{HR}$ and CI. A nonparametric bootstrap resampling procedure is also proposed for estimating both the standard error and the confidence intervals of the model coefficients [16]. HR is represented as SCL in ms, as in previous studies [9], [17].

Ridge Regression Data Model. Assuming a general form of the nonlinear regression model with $K+1$ terms,

$$
\widehat{T}=w_{0}+w_{1} \varphi_{1}+w_{2} \varphi_{2}+\cdots+w_{K} \varphi_{K}
$$

the independent variables $\varphi_{k}, k=1, \ldots, K$ represent the individual variables SCL, CI, and nonlinear combinations and powers of these variables, and $\widehat{T}$ represents a parameter assessing the HRT, which in this work is TS. If the model coefficients are arranged in a column vector $\boldsymbol{w}$, then the nonlinear regression model can be written as

$$
T_{i}=\boldsymbol{w}^{T} \boldsymbol{\varphi}_{i}+\varepsilon_{i}=\widehat{T}_{i}+\varepsilon_{i} ; \quad i=1, \ldots, N
$$

where $\varphi_{i}=\left[1, \varphi_{1, i}, \ldots, \varphi_{K, i}\right]^{T}$, and $N$ is the total number of VPCs. In matrix notation, (2) can be rewritten as

$$
\boldsymbol{T}=\boldsymbol{\Phi} \boldsymbol{w}+\varepsilon
$$

where $\quad \boldsymbol{\Phi}=\left[\begin{array}{llll}\boldsymbol{\varphi}_{1} & \boldsymbol{\varphi}_{2} & \ldots & \boldsymbol{\varphi}_{N}\end{array}\right]^{T}, \quad \boldsymbol{T}=\left[T_{1}, \ldots, T_{N}\right]^{T}$, $\boldsymbol{w}=\left[w_{0}, \ldots, w_{K}\right]^{T}$, and $\varepsilon=\left[\varepsilon_{1}, \ldots, \varepsilon_{N}\right]^{T}$ is the error term.

To compute $\boldsymbol{w}$, we used the least squares (LS) criterion modified by including a regularization term. This term provides a trade-off between bias and variance for the model [18], [19]. The influence of the regularization term is controlled by parameter $\lambda$,

$$
\min _{\boldsymbol{w}}\left\{\|\boldsymbol{T}-\boldsymbol{\Phi} \boldsymbol{w}\|_{2}^{2}+\lambda\|\boldsymbol{w}\|_{2}^{2}\right\}
$$




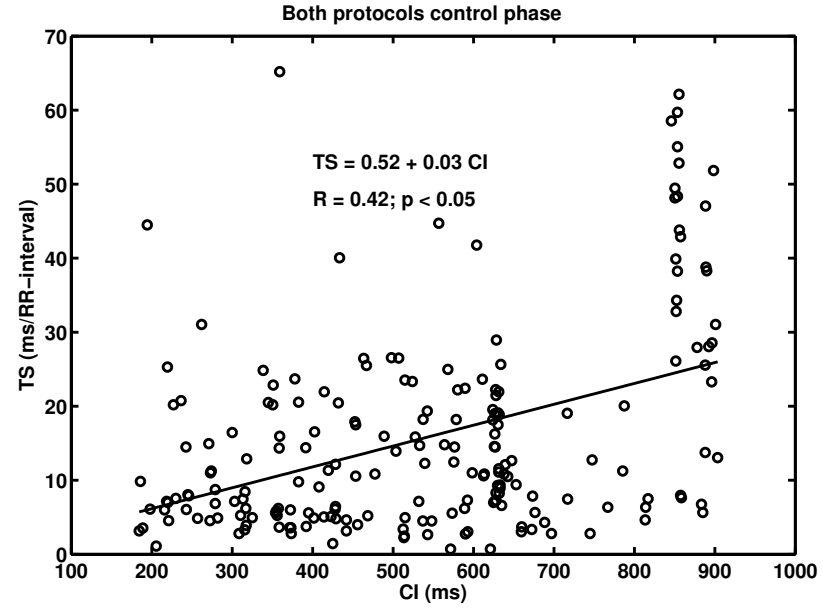

(a)

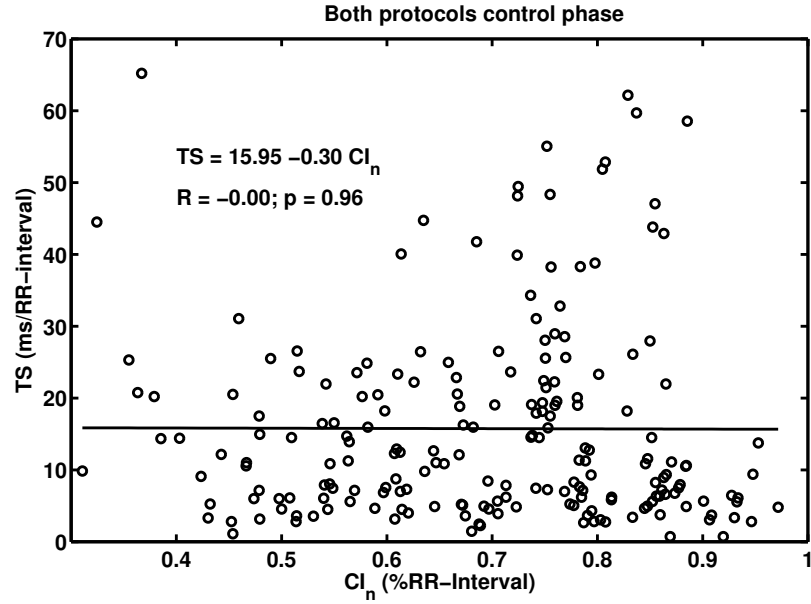

(b)

Fig. 1. Linear regression of TS vs CI, and vs $C I_{n}$, in a population of 11 patients with no structural heart disease from both protocols in the control phase (without isoproterenol) $(\mathrm{a}, \mathrm{b})$.

TABLE I

DIFFERENT NONLINEAR RIDGE REGRESSION MODELS ANALYZED.

\begin{tabular}{lc}
\hline \hline Model & Name \\
\hline \hline$\varphi=[C I]$ & $\mathrm{M}_{1}$ \\
$\varphi=\left[S C L, C I, S C L^{2}, C I^{2}, S C L \cdot C I\right]^{T}$ & $\mathrm{M}_{2}$ \\
$\varphi=\left[S C L, C I, S C L^{2}, S C L^{3}, C I^{2}, C I^{3}, S C L \cdot C I\right]^{T}$ & $\mathrm{M}_{3}$ \\
\hline \hline
\end{tabular}

The solution is given by

$$
\boldsymbol{w}=\left(\boldsymbol{\Phi}^{T} \boldsymbol{\Phi}+\lambda \mathbb{I}\right)^{-1} \boldsymbol{\Phi}^{T} \boldsymbol{T}
$$

where $\lambda$ is usually estimated using a cross-validation procedure, and $\mathbb{I}$ is the identity matrix [19].

Several regression models have been analyzed in this work. First, a linear regression model $\left(\mathrm{M}_{1}\right)$, using $C I$ as explanatory variable, allows us to replicate the results found in the literature on low noise EPS data. In order to isolate the effect of $\mathrm{CI}$ from the effect of HR, we also considered the normalized CI [10], defined as $\mathrm{CI}_{n}=\mathrm{CI} / R R_{-1}$, where $R R_{-1}$ denotes the RR interval preceding the VPC. Second, two nonlinear ridge regression models are proposed including quadratic $\left(\mathrm{M}_{2}\right)$ and cubic $\left(\mathrm{M}_{3}\right)$ powers of the explanatory variables, as well as a first order interaction term. The regression models are summarized in Table I. Since some of the variables are powers, or combinations, of SCL and CI, the ranges differ widely, and variables with large values could have too much influence on the cost function. To overcome this problem, data was modified by subtracting its mean and dividing by its standard deviation [20], so that variables can be presented in normalized units (n.u.).

Two TS values were computed for each patient in variable CI protocol, both in control and in isoproterenol phase, using the classical approach of averaging a minimum of 5 tachograms grouped according to short and long CI.

Model Performance. The accuracy of the nonlinear regression model was assessed using cross-validation and obtaining the mean squared error (MSE),

$$
M S E=\frac{1}{N} \sum_{i=1}^{N}\left(\widehat{T}_{i}-T_{i}\right)^{2}
$$

and the $R^{2}$ statistic,

$$
R^{2}=1-\frac{R S S}{T S S}
$$

where $R S S=N \cdot M S E$ is the residual sum of squares, $T S S=\sum_{i=1}^{N}\left(T_{i}-\bar{T}\right)^{2}$ is the total sum of squares, and $\bar{T}$ is the average of all $T_{i}$ values. The parameter $R^{2}$ measures the fraction of the total variance of TS that is explained by the model variables, and varies between 0 and 1 . We applied a 10-fold cross-validation procedure, often used in the literature [21]. In this procedure, the dataset is randomly divided into 10 groups of equal size, named folds, and 10 nonlinear regression models are constructed. Each model is fitted by using data from nine-folds, while the remaining fold is used as a validation set to compute the accuracy measures. This process is repeated 10 times, so that the validation set corresponds to a different fold every time. The final estimation of the measures is computed by averaging the results obtained for the 10 validation sets.

Bootstrap Procedure. Empirical distributions of the coefficients of the nonlinear ridge regression model were computed by using a nonparametric resampling procedure. Bootstrapping is a powerful statistical tool that emulates the process of obtaining new datasets by resampling of an existing dataset with replacement [16]. It allows us to obtain coefficient estimates from different datasets by repeatedly resampling observations from the original dataset [21].

Let us denote an observation as the pair $\left(T_{i}, \varphi_{i}\right)$, where $i=1, \ldots, N$, and $N$ is the number of observations in the original dataset. Therefore, the complete original data set is $Z=(\boldsymbol{T}, \boldsymbol{\Phi})$. The bootstrap procedure consists of randomly selecting $N$ observations with replacement from $Z$ to obtain a bootstrap dataset $Z^{* 1}$. Since resampling is performed with replacement, a given observation can be included more than 


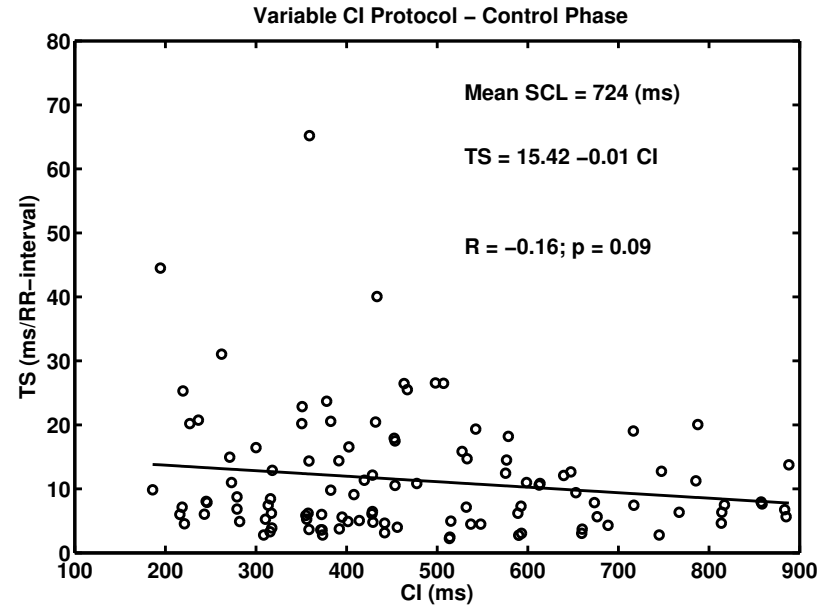

(a)

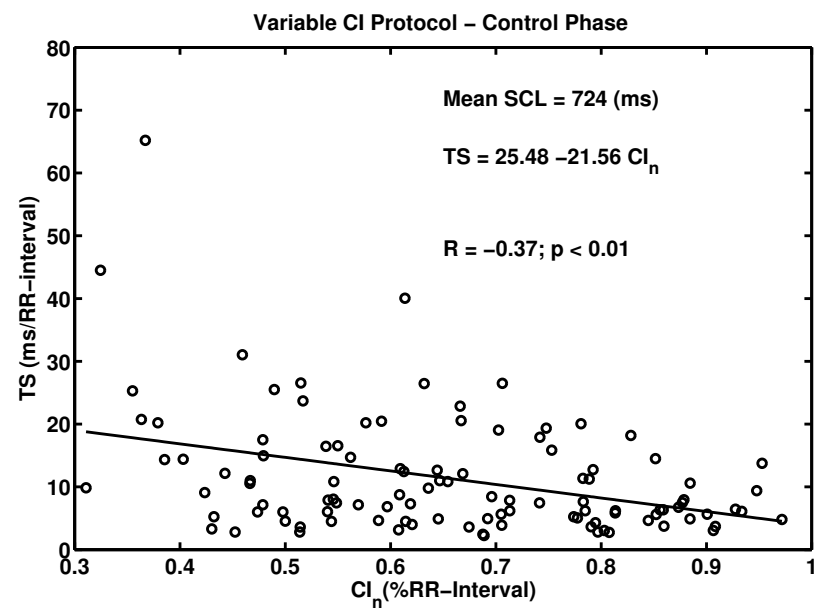

(c)

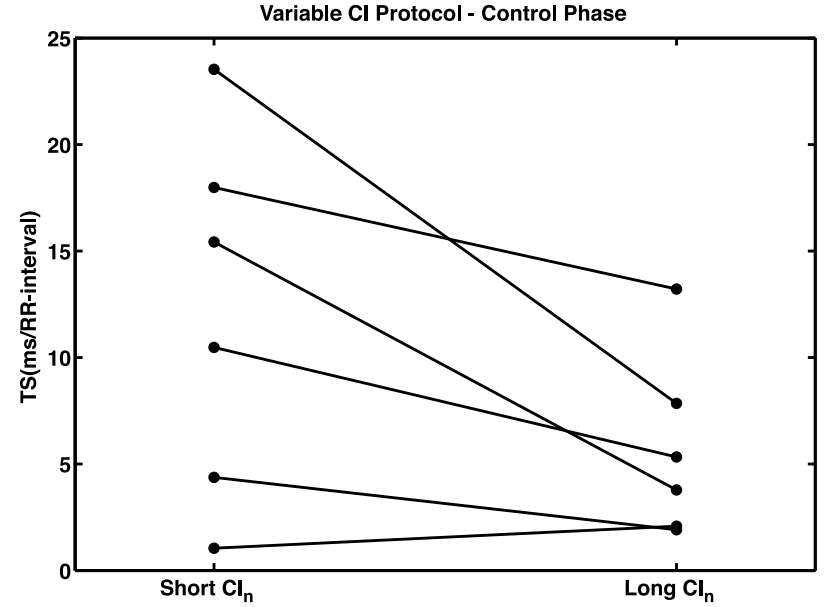

(e)

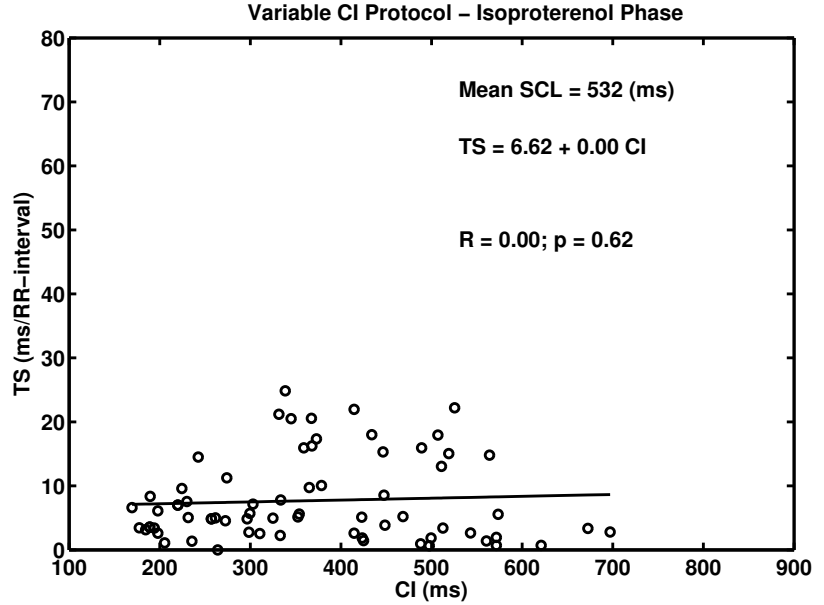

(b)

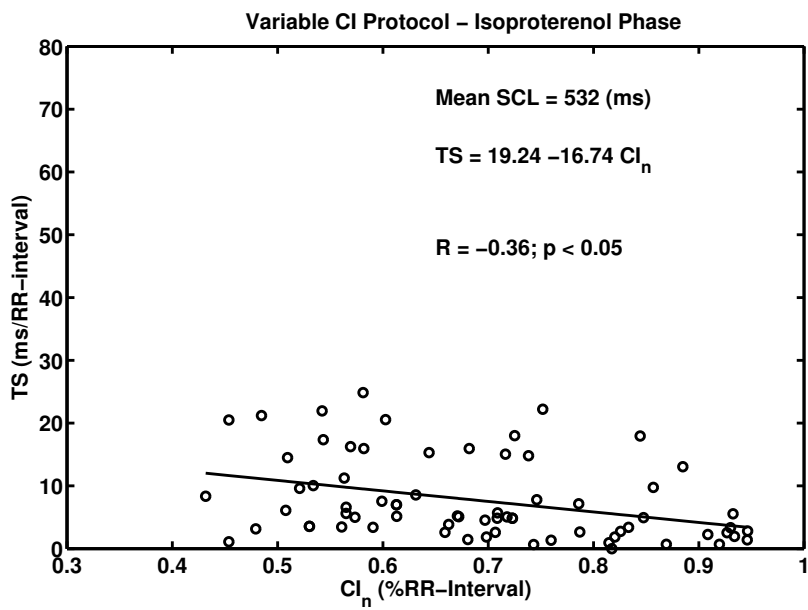

(d)

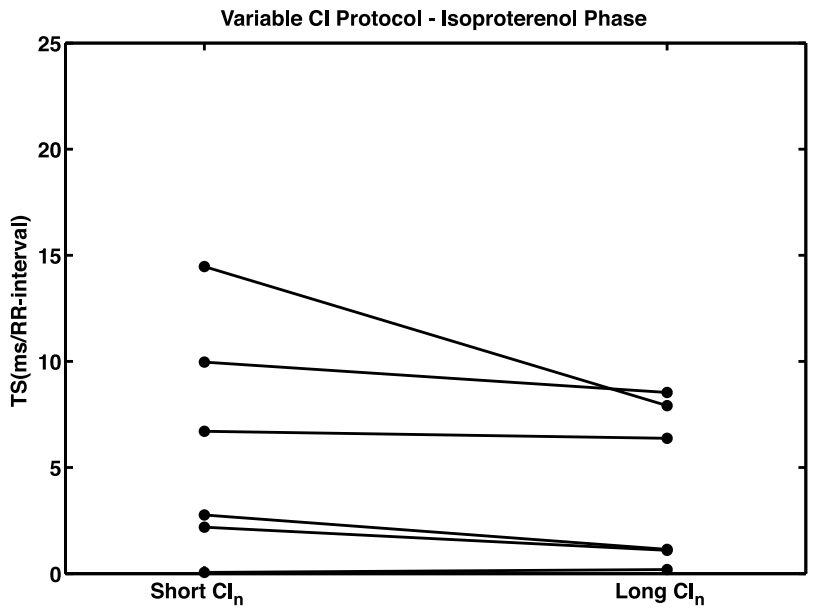

(f)

Fig. 2. Analysis of the interaction between HR and CI using data from patients in variable CI protocol, control phase (a,c), and isoproterenol phase (b,d). Mean and standard deviation of SCL for each phase are reported. Interaction between HR and CI using TS computed from an averaged tachogram grouped by $C I_{n}$, control phase (e) and isoproterenol phase (f). Solid lines connect TS values from the same subject. 
once in $Z^{* 1}$. Coefficients are estimated using $Z^{* 1}$, leading to a bootstrap estimate for $\boldsymbol{w}$, called $\hat{\boldsymbol{w}}^{* 1}$. This procedure is repeated $B$ times to produce $B$ bootstrap datasets, $\left\{Z^{* i}\right\}_{i=1}^{B}$, and accordingly, to produce $B$ bootstrap estimates of the coefficients $\left\{\hat{\boldsymbol{w}}^{* i}\right\}_{i=1}^{B}$ [21].

From those estimated distributions, it is straightforward to test the null hypotheses for each of the coefficients in the model. We test the null hypothesis that parameter $w_{j}=0$, meaning that the associated variable does not explain TS. The alternative hypothesis is that $w_{j} \neq 0$, meaning that the corresponding variable is relevant, i.e. there is a linear relationship between variable and response. This can be stated as follows:

$$
\left\{\begin{array}{l}
H_{0}: w_{j}=0 \\
H_{1}: w_{j} \neq 0
\end{array}\right.
$$

This hypothesis test can be readily performed from the bootstrap empirical distribution of parameter $w_{j}$. The null hypothesis $H_{0}$ is rejected if the $95 \%$ confidence interval of the parameters does not contain the zero value.

\section{RESUlts}

First, the linear regression model, $\mathrm{M}_{1}$ is fitted to the EPS database control phase (i.e., without isoproterenol), aiming to reproduce the conclusions in the literature [9]. Second, linear regression models are fitted by using data separated into fixed CI and variable CI protocol, aiming to check whether the design of the protocol allows us to determine the influence of CI on HRT. Finally, nonlinear ridge regression models are fitted to the EPS and AMI databases to explain the influence of HR and CI on HRT.

TS as a Linear Function of CI. To compare with results in the literature, we analyzed the EPS database using $\mathrm{M}_{1}$, which was fitted using control phase patients of both the fixed and variable CI protocols.

Figure 1(a) shows a positive relationship between CI and TS that is statistically significant (given by $\mathrm{TS}=0.52+0.03 \mathrm{CI}$, $R=0.42, p<0.05$ ). This result is the opposite of what is expected from the baroreflex hypothesis. We found no correlation between $\mathrm{TS}$ and $\mathrm{CI}_{n}(R=-0.06, n . s$.$) in agreement$ with results in [9], see Figure 1(b).

TS as a Linear Function of CI and Interaction Effect of HR. The two phases, control and isoproterenol, of the variable CI protocol allowed us to study the effect of SCL on CI, by fitting $\mathrm{M}_{1}$ for each of the phases.

Figure 2 represents the results of simple linear regression models, $\mathbf{M}_{1}$, for control and isoproterenol phases, showing no significant relationship $(p>0.05)$ between TS and CI ( $R=-0.16$ and $R=-0.006$, respectively). However, using $\mathrm{CI}_{n}$ yielded TS models with significant negative slope, given by TS $=25.48-21.56 \mathrm{CI}_{n}$, with $R=-0.37, p<0.01$, and by TS $=19.24-16.74 \mathrm{CI}_{n}$ with $R=-0.36, p<0.05$. This behavior is consistent with the HRT baroreflex hypothesis. Moreover, the slope coefficient was lower with high HR (low SCL), hence proving an interaction effect of HR on CI according to other previous results [14]. Figures 2(e) and 2(f) show individual TS values calculated from averaged tachograms from the six patients in the variable CI protocol. Similar to the graphs above them, higher $\mathrm{CI}_{n}$ produces smaller $\mathrm{TS}$, and the negative relationship between $\mathrm{CI}$ and TS is damped at higher HR, achieved with isoproterenol.

$T S$ as a nonlinear function of CI and HR. Next, we present the results after fitting nonlinear regression models $\mathrm{M}_{2}$ and $\mathrm{M}_{3}$ to EPS and AMI databases. Table II and Figure $3\left(\mathrm{M}_{3}\right.$ only) show the coefficient values of the nonlinear models fitted to the EPS data with and without isoproterenol and the low and high mortality risk AMI data. In $\mathrm{M}_{3}$ and for the EPS without isoproterenol and AMI low-risk databases, the CI coefficient was significantly, and negatively, correlated with TS, in agreement with the hypothesis of baroreflex source of HRT. Coefficients relating SCL and TS were significant, and positive, for $\mathrm{M}_{2}$ and $\mathrm{M}_{3}$. Interaction term SCL.CI was also significant for $\mathrm{M}_{2}$ and $\mathrm{M}_{3}$. Models $\mathrm{M}_{2}$ and $\mathrm{M}_{3}$ fitted to EPS with isoproterenol dataset obtained the highest value of $R^{2}$, since TS for these subjects had a small variability around the fit line due to the high basal HR.

The model coefficients of $\mathrm{M}_{2}$ and $\mathrm{M}_{3}$, fitted to the AMI high-risk database, exhibited a relationship between TS and CI that was completely different from that obtained when analyzing the EPS and AMI low-risk databases. Figure 3 shows the mean and $95 \%$ confidence intervals, as estimated with bootstrap resampling, for the coefficients of nonlinear ridge regression model $\mathrm{M}_{3}$ fitted to EPS control (Figure 3(a)) and isoproterenol phase (Figure 3(b)), AMI low-risk, and AMI high-risk database. Changes in the relationship between TS and $\mathrm{CI}$ due to the interaction of the HR can be observed in Figure 3(b). In the fitted model $\mathrm{M}_{3}$ for EPS patients with high $\mathrm{HR}$, isoproterenol phase, none of the coefficients related to $\mathrm{CI}$ was significant. The SCL*CI term was significant in all the models except $\mathrm{M}_{2}$ for AMI high-risk. The inclusion of this interaction term in the model may decouple the effect of HR on CI.

\section{Discussion}

In this work, HRT parameters were computed on single VPC tachograms. They were also compared with the classical procedure of averaging a number of individual tachograms. Previous works focused on methods for reliable estimation of HRT parameters from individual VPC responses. For instance, HRT statistical detection was addressed by using an extension of the integral pulse frequency modulation model accounting for HRT [22], [23], [24]. In this approach, the tachogram was assumed to reflect the combination of heart rate variability (modeled as white Gaussian noise) and HRT (modeled as a linear combination of Karhunen-Loève basis functions). Another approach consisted of filtering individual VPC tachograms by using a robust method based on support vector machines [4].

Several physiological factors modulate the HRT pattern [6], [7], [8]. The dependence of HRT on HR is attributed to a shared sympathovagal modulation, i.e. HRT is attenuated at high HR conditions. Although some studies support this physiological hypothesis by showing a strong correlation between HR and HRT across individuals [25], [17], only weak correlation was found in individual subjects [3]. In [26], the CI was found to be correlated only with TO, but TS was 
TABLE II

COEFFICIENT VALUES OF NONLINEAR RIDGE REGRESSION MODELS FITTED TO EPS DATABASE. STATISTICALLY SIGNIFICANT VARIABLES ARE HIGHLIGHTED AND DENOTED BY* * SYMBOL - MEANS THAT THE VARIABLE WAS NOT INCLUDED IN THE MODEL.

\begin{tabular}{|c||c|c|c|c|c|c|c||c|c|}
\hline EPS Control & $S C L$ & $C I$ & $S C L^{2}$ & $S C L^{3}$ & $C I^{2}$ & $C I^{3}$ & $S C L \cdot C I$ & MSE & $R^{2}$ \\
\hline \hline $\mathrm{M}_{2}$ & $\mathbf{2 . 0 8}^{*}$ & -0.65 & $\mathbf{3 . 0 1}^{*}$ & - & 0.76 & - & $\mathbf{1 . 7 0}^{*}$ & 145.41 & 0.22 \\
$\mathrm{M}_{3}$ & $\mathbf{1 . 3 6}^{*}$ & $\mathbf{- 1 . 0 2}^{*}$ & $\mathbf{2 . 0 5}^{*}$ & $\mathbf{2 . 7 4}^{*}$ & -0.13 & $\mathbf{1 . 1 1}^{*}$ & $\mathbf{0 . 9 1}^{*}$ & 149.67 & 0.20 \\
\hline \hline EPS ISO & $S C L$ & $C I$ & $S C L^{2}$ & $S C L^{3}$ & $C I^{2}$ & $C I^{3}$ & $S C L \cdot C I$ & MSE & $R^{2}$ \\
\hline \hline $\mathrm{M}_{2}$ & $\mathbf{1 . 3 2}^{*}$ & 0.06 & $\mathbf{1 . 6 9}^{*}$ & - & $\mathbf{0 . 5 2}^{*}$ & - & $\mathbf{1 . 0 5}^{*}$ & 52.23 & 0.33 \\
$\mathrm{M}_{3}$ & $\mathbf{1 . 0 1}^{*}$ & -0.09 & $\mathbf{1 . 2 5}^{*}$ & $\mathbf{1 . 3 6}^{*}$ & 0.23 & 0.45 & $\mathbf{0 . 6 8}^{*}$ & 52.65 & 0.32 \\
\hline \hline AMI low-risk & $S C L$ & $C I$ & $S C L^{2}$ & $S C L^{3}$ & $C I^{2}$ & $C I^{3}$ & $S C L \cdot C I$ & MSE & $R^{2}$ \\
\hline \hline $\mathrm{M}_{2}$ & $\mathbf{3 . 4 4}^{*}$ & $\mathbf{- 1 . 5 5}^{*}$ & $\mathbf{3 . 3 2}^{*}$ & - & $\mathbf{- 1 . 8 3}^{*}$ & - & $\mathbf{0 . 8 2}^{*}$ & 300.93 & 0.13 \\
$\mathrm{M}_{3}$ & $\mathbf{2 . 6 5}^{*}$ & $\mathbf{- 1 . 0 5}^{*}$ & $\mathbf{2 . 4 9}^{*}$ & $\mathbf{2 . 2 8}^{*}$ & $\mathbf{- 1 . 2 6}^{*}$ & $\mathbf{- 1 . 4 3}^{*}$ & $\mathbf{0 . 6 3}^{*}$ & 297.20 & 0.15 \\
\hline \hline AMI high-risk & $S C L$ & $C I$ & $S C L^{2}$ & $S C L^{3}$ & $C I^{2}$ & $C I^{3}$ & $S C L \cdot C I$ & MSE & $R^{2}$ \\
\hline \hline $\mathrm{M}_{2}$ & $\mathbf{1 . 1 3}^{*}$ & $\mathbf{1 . 6 3}^{*}$ & $\mathbf{- 0 . 6 9}^{*}$ & - & 0.14 & - & 0.02 & 16.77 & 0.21 \\
$\mathrm{M}_{3}$ & $\mathbf{1 . 1 8}^{*}$ & $\mathbf{1 . 4 7}^{*}$ & 0.08 & $\mathbf{- 1 . 1 2}^{*}$ & $\mathbf{0 . 5 9}^{*}$ & -0.58 & $\mathbf{0 . 5 7}^{*}$ & 16.82 & 0.20 \\
\hline
\end{tabular}

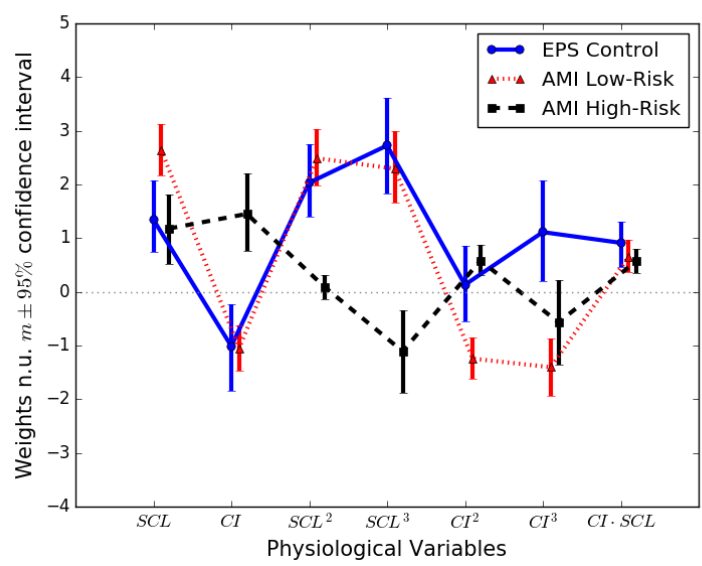

(a)

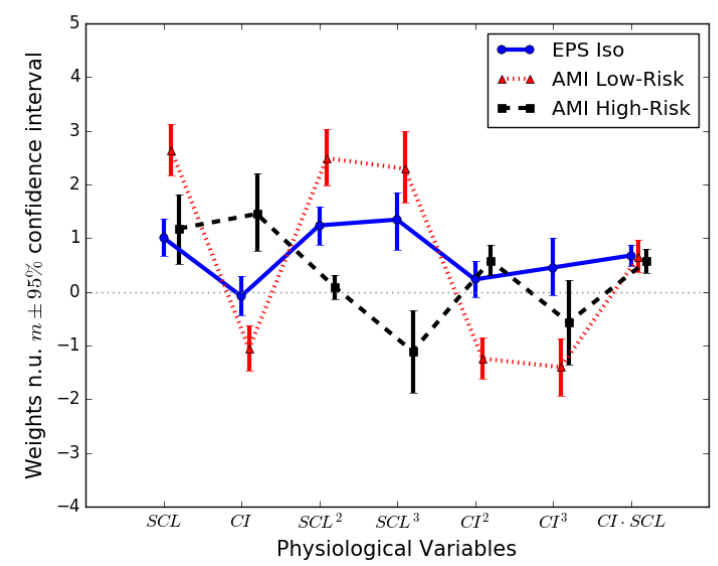

(b)

Fig. 3. Coefficient values (mean and the $95 \%$ confidence interval) of nonlinear ridge regression model $\mathrm{M}_{3}$ fitted to EPS (control phase (a), isoproterenol phase(b)), AMI high-risk, and AMI low-risk databases.

not affected at all, whereas [11] and [27] reported strong correlations of both TO and TS with CI. Interestingly, this effect was less pronounced in patients with left ventricular dysfunction [11]. However, no correlation between HRT parameters and CI was found in [9], neither in a pooled population nor in individual patients. Conflicting results on correlation between HRT parameters and CI have usually been attributed to the effect of basal HR. According to [28], HRT response is severely attenuated when HR is high. In this case, HRT parameters are unlikely to be correlated with CI.

There have been some attempts to study the joint influence of CI and HR on HRT by representing TS and TO as a function of CI and HR [12], however, no clear pattern could be observed. In our case, normalization was found to decouple the effect of SCL on CI by using only data from patients in variable CI protocol control phase, revealing the expected physiological modulation of CI on TS. The results in [9] suggested that correlation was due to strong influence of HR on HRT (low HR-high TS, and high HR-low TS), rather than an inherent relationship between HRT and CI. However, no further analysis was presented therein. In our results, nonlinear model $\mathrm{M}_{3}$ showed a relationship between CI and HRT which is in agreement with the baroreflex hypothesis, both in EPS control phase subjects, and in AMI low-risk patients. Interestingly, in EPS isoproterenol phase subjects, i.e. high HR, all the coefficients related to CI were statistically non significant. These results may confirm the suggestion that high HR distorts the relationship between CI and HRT parameters.

\section{CONCLUSiOnS}

In this work, we have proposed a procedure to systematically assess the modulation of HRT by CI and HR. Using this approach, significant correlation was found between these physiological factors and the TS, thus supporting the baroreflex source of HRT. The proposed nonlinear model accounted for the effect of SCL and CI simultaneously. It was able to attribute weights (coefficients) that are in agreement with the physiological baroreflex explanation of HRT.

With the approach proposed in this work, two main results are obtained: First, a quantification of the effect of coupling 
interval on TS, which supports the hypothesis of the baroreflex origin of HRT; and second, a nonlinear model that explains the modulation of HRT by HR and CI.

\section{ACKNOWLEDGMENT}

This work was partially supported by the Spanish Government under Research Projects TEC2013-48439-C4-1-R, TEC2013-48439-C4-2-R, TEC2013-42140-R, and TIN201453567-R.

\section{REFERENCES}

[1] G. Schmidt, M. Malik, P. Barthel, R. Schneider, K. Ulm, L. Rolnitzky, A. J. Camm, J. T. Bigger, and A. Schömig, "Heart-rate turbulence after ventricular premature beats as a predictor of mortality after acute myocardial infarction," Lancet, vol. 353, no. 9162, pp. 1390-1396, 1999.

[2] A. Bauer, M. Malik, G. Schmidt, P. Barthel, H. Bonnemeier, I. Cygankiewicz, P. Guzik, F. Lombardi, A. Müller, A. Oto, R. Schneider, M. Watanabe, D. Wichterle, and W. Zareba, "Heart rate turbulence: standards of measurement, physiological interpretation, and clinical use: International Society for Holter and Noninvasive Electrophysiology Consensus," J. Am. Coll. Cardiol., vol. 52, no. 17, pp. 1353-1365, 2008.

[3] M. A. Watanabe and G. Schmidt, "Heart rate turbulence: a 5-year review," Heart Rhythm, vol. 1, no. 6, pp. 732-738, 2004.

[4] J. L. Rojo Alvarez, O. Barquero-Pérez, I. Mora-Jiménez, E. Everss, A. B. Rodríguez-González, and A. García Alberola, "Heart rate turbulence denoising using support vector machines." IEEE Trans. Biomed. Eng., vol. 56, no. 2, pp. 310-319, 2009.

[5] O. Barquero-Pérez, C. Figuera-Pozuelo, R. Goya-Esteban, I. MoraJiménez, J. Rojo-Alvarez, J. Gimeno-Blanes, and A. Garcia-Alberola, "Heart rate turbulence modulation with coupling interval and heart rate," Comput. Cardiol., vol. 39, pp. 173-176, 2012.

[6] A. Bauer, M. Malik, P. Barthel, R. Schneider, M. A. Watanabe, A. J. Camm, A. Schömig, and G. Schmidt, "Turbulence dynamics: an independent predictor of late mortality after acute myocardial infarction," Int. J. Cardiol., vol. 107, no. 1, pp. 42-47, 2006.

[7] J. Francis, M. A. Watanabe, and G. Schmidt, "Heart rate turbulence: a new predictor for risk of sudden cardiac death," Ann. Noninvasive Electrocard., vol. 10, no. 1, pp. 102-109, 2005.

[8] M. Watanabe, M. Alford, R. Schneider, A. Bauer, P. Barthel, P. Stein, and G. Schmidt, "Demonstration of circadian rhythm in heart rate turbulence using novel application of correlator functions," Heart Rhythm, vol. 4, no. 3, pp. 292-300, 2007.

[9] M. A. Watanabe, J. E. Marine, R. Sheldon, and M. E. Josephson, "Effects of ventricular premature stimulus coupling interval on blood pressure and heart rate turbulence," Circulation, vol. 106, no. 3, pp. 325-330, 2002.

[10] A. Bauer, P. Barthel, R. Schneider, and M. Malik, "Impact of coupling interval on heart rate turbulence," Eur. Heart J., vol. 22, p. 438, 2001.

[11] I. Savelieva, D. Wichterle, M. Harries, M. Meara, A. J. Camm, and M. Malik, "Heart rate turbulence after atrial and ventricular premature beats: relation to left ventricular function and coupling intervals," Pacing Clin. Electrophysiol. (PACE), vol. 26, no. 1 Pt 2, pp. 401-405, 2003.

[12] G. Schmidt, A. Bauer, R. Schneider, P. Barthel, M. Malik, A. J. Camm, and A. Schömig, "Heart rate turbulence: Impact of coupling interval and preceding sinus interval," Eur. Heart. J., vol. 21, no. Suppl, p. 552, 2000.

[13] S. Steinberg, R. Robinson, and M. Rosen, "Molecular and cellular bases of $\beta$-adrenergic and $\alpha$-adrenergic modulation of cardiac rhythm," in Cardiac Electrophysiology: From Cell to Bedside, D. Zipes and J. Jalife, Eds. WB Saunders, 2005.

[14] A. Voss, V. Baier, A. Schirdewan, and U. Leder, "Physiological hypotheses on heart rate turbulence," in Dynamic Electrocardiography, M. Malik and A. J. Camm, Eds. Blackwell Publishing, 2007, pp. 203-210.

[15] J. González-Carrillo, A. García-Alberola, D. Saura, P. Carrillo, R. López, J. J. Sánchez-Muñoz, J. Martínez, and M. Valdés, "Impacto de la angioplastia primaria en la indicación de desfibrilador implantable en pacientes con infarto de miocardio," Resv. Esp. Cardiol., vol. 56, no. 12, pp. 52-56, 2003.

[16] B. Efron and R. J. Tibshirani, An Introduction to the Bootstrap. Chapman \& Hall, 1993.
[17] J. Schwab, G. Eichner, N. Shlevkov, J. Schrickel, A. Yang, O. Balta, T. Lewalter, and B. Lüderitz, "Impact of age and basic heart rate on heart rate turbulence in healthy persons," Pacing Clin. Electrophysiol. (PACE), vol. 28, pp. S198-S201, 2005.

[18] C. M. Bishop, Pattern Recognition and Machine Learning (Information Science and Statistics). Springer, 2007.

[19] T. Hastie, R. Tibshirani, and J. Friedmann, The Elements of Statistical Learning. Data Mining, Inference, and Prediction. New York: Springer, 2001.

[20] S. Theodoridis and K. Koutroumbas, Pattern Recognition, 4th ed. Academic Press, 2008.

[21] G. James, T. Hastie, D. Witten, and R. Tibshirani, An Introduction to Statistical Learning: With Applications in R. Springer London, Limited, 2013.

[22] K. Solem, P. Laguna, J. P. Martínez, and L. Sörnmo, "Model-based detection of heart rate turbulence." IEEE Trans. Biomed. Eng., vol. 55, no. 12, pp. 2712-2722, 2008.

[23] D. Smith, K. Solem, P. Laguna, J. P. Martínez, and L. Sörnmo, "Modelbased detection of heart rate turbulence using mean shape information." IEEE Trans. Biomed. Eng., vol. 57, no. 2, pp. 334-342, 2010.

[24] J. P. Martínez, I. Cygankiewicz, D. Smith, A. Bayes de Luna, P. Laguna, and L. Sörnmo, "Detection performance and risk stratification using a model-based shape index characterizing heart rate turbulence." Ann. Biomed. Eng., vol. 38, no. 10, pp. 3173-3184, 2010.

[25] A. Bauer, R. Schneider, P. Barthel, M. Malik, and G. Schmidt, "Heart rate turbulence dynamicity," Eur. Heart J., vol. 22, no. Abstr. Suppl, p. 436, 2001.

[26] J. O. Schwab, N. Shlevkov, K. Grunwald, J. W. Schrickel, A. Yang, L. Lickfett, T. Lewalter, and B. Lüderitz, "Influence of the point of origin on heart rate turbulence after stimulated ventricular and atrial premature beats." Basic Res. Cardiol., vol. 99, no. 1, pp. 56-60, 2004.

[27] K.-T. Lee, W.-T. Lai, C.-S. Chu, H.-W. Yen, W.-C. Voon, and S.-H. Sheu, "Effect of electrophysiologic character of ventricular premature beat on heart rate turbulence." J. Electrocardiol., vol. 37, no. 1, pp. 41-46, 2004.

[28] M. A. Watanabe, "Heart rate turbulence: a review." Indian Pacing Electrophysiol. J., vol. 3, no. 1, pp. 10-22, 2003.

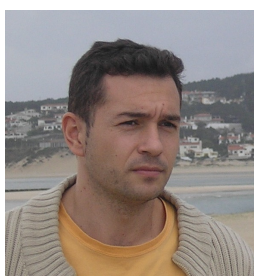

Óscar Barquero-Pérez received the Tech. Telecom. Eng. degree in technical telecommunication engineering from Universidad Carlos III de Madrid, Madrid, Spain, in 2005, and the M.Sc. degree in biomedical engineering from Universidade do Porto, Portugal, in 2008, and Ph.D. degree from Universidad Rey Juan Carlos, Madrid, Spain, in 2014. Since 2007, he has been with the Department of Signal Theory and Communications, Universidad Rey Juan Carlos, Madrid. His current research interests include nonlinear time-series analysis, biomedical signal processing and statistical learning.

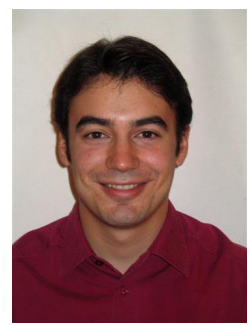

Carlos Figuera received the Telecommunication Engineering Degree in 2002 from Polytechnic University of Madrid, Spain. He received the $\mathrm{PhD}$ degree on 2009. He is currently working as an assistant professor in the Department of Signal Theory and Communications at Rey Juan Carlos University in Madrid, Spain. His research interests include signal processing for wireless communications and statistical learning theory with application to biological signal processing. 


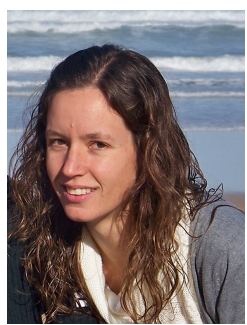

Rebeca Goya-Esteban received the BSc degree in Telecommunication Engineering from Carlos III University, Madrid, Spain, in 2006, the MSc degree in Biomedical Engineering from Porto University, Portugal, in 2008 and the $\mathrm{PhD}$ degree in Telecommunications Engineering from Rey Juan Carlos University, Madrid, Spain, in 2014. Currently, she is visiting lecturer and researcher at Rey Juan Carlos University. Her main research interests include time series analysis, cardiac signal processing and statistical learning.

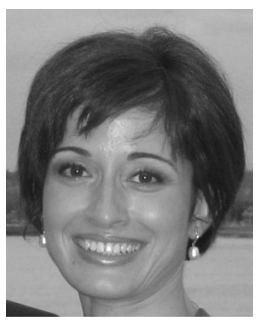

Inmaculada Mora-Jiménez received the Telecommunication Engineering degree from the Universidad Politecnica de Valencia, Valencia, Spain, in 1998, and the Ph.D. degree from the Universidad Carlos III de Madrid, Madrid, Spain, in 2004. She is currently an Associate Professor in the Department of Signal Theory and Communications, Universidad Rey Juan Carlos, Madrid, Spain. Her main research interests include statistical learning theory, neural networks, and their applications to image processing, bioengineering, and communications.

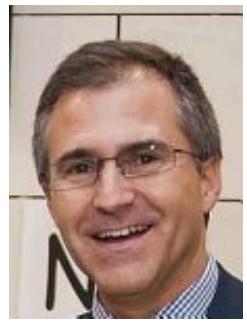

Francisco J. Gimeno-Blanes Telecommunications engineer, Advanced Studies in Taxation and Business Administration and Ph.D. in Communications Technologies. He is currently Professor at the University Miguel Hernández, ViceDean of the Polytechnic School of Elche, and Adjunct VicePresident for Technology Development. He has worked in telecom industry for 10 years holding management positions as Director of Strategic Planning, responsible for Strategic Planning at Telefónica DataCorp, member of the Chairman's Office of Telefónica Holding and Corporate Development at Grupo Fuertes. Primary research lines are in the Biosignals processing, business planning, business valuation, financial risk management, pricing and marketing forecasting.

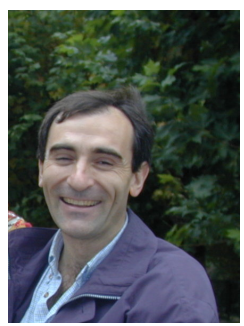

Pablo Laguna is Full Professor of Signal Processing and Communications in the Department of Electrical Engineering at the Engineering School, where he has being vice-dean for international relation (19992002), and a researcher at the Aragón Institute for Engineering Research (I3A), both at University of Zaragoza, Spain, where he has being responsible of the Biomedical Engineering division of the I3A (2000-2011) and of the master in Biomedical Engineering (2003-2010). He is also member, and has served as scientific director (2011-2015), of the Spanish Center for Biomedical Engineering, Biomaterial and Nano-medicine Research CIBER-BBN. His professional research interests are in Signal Processing, in particular applied to Biomedical applications. He has co-authored more than 130 research papers on this topic, over 250 international conference papers, and has advise $13 \mathrm{Ph} . \mathrm{D}$ Thesis. He has lead a broad number of projects on biomedical signal interpretation specially in the cardiovascular domain, most of them with international collaborations at clinical and engineering sites. He is having some international scientific responsibilities, as serving as president the board of directors of Computing in Cardiology conference, editor of the digital signal processing journal (Eurasip), and of the Medical and Biological Engineering and Computing, organizer of different scientific conferences, etc. He is also responsible of the Ph.D. program in Biomedical Engineering at Zaragoza University. He is, together with L. Sörnmo, the author of Bioelectrical Signal Processing in Cardiac and Neurological Applications, book (Elsevier, 2005)

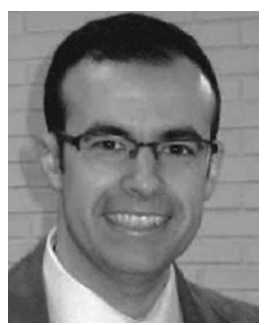

Juan Pablo Martínez was born in Zaragoza, Spain, in 1976. He received the M.S. degree in telecommunication engineering and the Ph.D. degree in biomedical engineering from the University of Zaragoza (UZ), Zaragoza, in 1999 and 2005, respectively. In 2000, he was an Assistant Professor at the Aragon Institute of Engineering Research, UZ, where since 2007, he has been an Associate Professor. He is also with the Centro de Investigación Biomédica en Red en Bioingeniería, Biomateriales y Nanomedicina, Zaragoza. His current research interests include biomedical signal processing, with main interest in signals of cardiovascular origin.

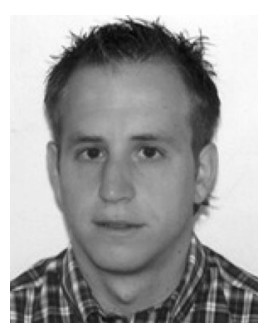

Eduardo Gil was born in Zaragoza, Spain, in 1978. He received the M.S. degree in telecommunication engineering and the $\mathrm{Ph}$. D. degree in biomedical engineering from the University of Zaragoza, Zaragoza, Spain, in 2002 and 2009, respectively, and the Master's degree "Master universitario en Sueño: Fisiología y Medicina" from the University Pablo Olavide, Sevilla, Spain, in 2007. Since 2006, he has been an Assistant Professor with the University of Zaragoza, where he is currently a Researcher with the Aragon Institute for Engineering Research and also with the Biomedical Research Networking Center in Bioengineering, Biomaterials, and Nanomedicine. His current research interests include biomedical signal processing, especially in the analysis of the photopletismography signal.

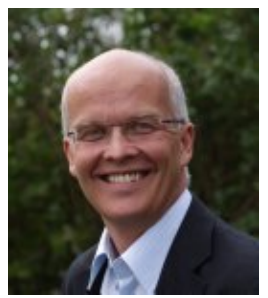

Leif Sörnmo (S'80-M'85-SM'02) received the M.Sc. and Ph.D. degrees in electrical engineering from Lund University, Lund, Sweden, in 1978 and 1984, respectively. From 1983 to 1995, Dr. Sörnmo was a Research Fellow at the Department of Clinical Physiology, Lund University, pursuing research in ECG signal processing. Since 1990, he has been with the Biomedical Signal Processing Group, Department of Biomedical Engineering, Lund University, where he is currently a Professor and responsible for the BME program. He is, together with Pablo Laguna, the author of Bioelectrical Signal Processing in Cardiac and Neurological Applications (Elsevier, 2005). His research interests include statistical signal processing, modeling of biomedical signals, methods for analysis of atrial fibrillation, multimodal signal processing in hemodialysis, and powerefficient signal processing in implantable devices. Dr. Sörnmo is a Fellow of International Academy of Medical and Biological Engineering and European Alliance for Medical and Biological Engineering. He is Associate Editor of IEEE TRANSACTIONS ON BIOMEDICAL ENGINEERING, Journal of Electrocardiology, and Medical and Biological Engineering \& Computing; he is on the editorial board of Journal of Biomedical Engineering. He was an Associate Editor of Computers in Biomedical Research (1997-2000). He is founder and director of the undergraduate and graduate program in biomedical engineering at Lund University. He serves on the board of the Computing in Cardiology conference.

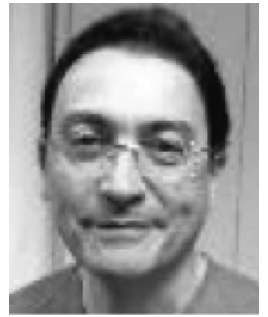

Arcadi García-Alberola received the M.D. and the $\mathrm{Ph}$.D. degrees from Universitat de Valencia, Burjassot, Spain, in 1982 and 1991, respectively. Since 1993, he has been a Cardiologist and a Professor of Medicine at Hospital Universitario Virgen de la Arrixaca and Universidad de Murcia, Murcia, Spain, where he is currently the Director of the Arrhythmia Unit of Cardiac Electrophysiology. He has coauthored more than 120 scientific papers and more than 50 communications in cardiac electrophysiology, and his main research areas are repolarization analysis, arrhythmia mechanisms, and cardiac signal processing. 


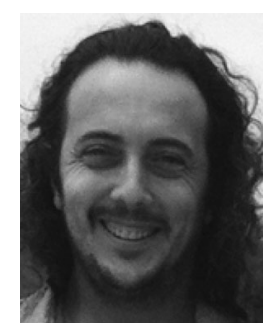

José Luis Rojo-Álvarez (SM12) received the Telecommunications Engineer degree (1996, University of Vigo), and the Ph.D. in Telecommunications (2000, Polytechnic University of Madrid). Since 2016, he has been Full Professor in Rey Juan Carlos University, Madrid, Spain. In 2015, he joined Persei vivarium as Chief Scientific Officer for building bridges between eHealth industry and academy research. He has published more than 85 papers in JCR journals and more than 150 conference communications. He has participated in more than 50 projects (with public and private funding). He received in 2009 the I3 Program to the Excellent Research Trajectory from Spanish Ministry of Science. His research interests include statistical learning and digital signal processing with applications to biomedical engineering and marketing. 